\title{
OPEN i-RheoFT: Fourier transforming sampled functions without artefacts
}

\begin{abstract}
Matthew G. Smith ${ }^{1}$, Graham M. Gibson ${ }^{2}$ \& Manlio Tassieri ${ }^{1 凶}$
In this article we present a new open-access code named "i-RheoFT" that implements the analytical method first introduced in [PRE, 80, 012501 (2009)] and then enhanced in [New J Phys 14, 115032 (2012)], which allows to evaluate the Fourier transform of any generic time-dependent function that vanishes for negative times, sampled at a finite set of data points that extend over a finite range, and need not be equally spaced. I-RheoFT has been employed here to investigate three important experimental factors: (i) the 'density of initial experimental points' describing the sampled function, (ii) the interpolation function used to perform the "virtual oversampling" procedure introduced in [New J Phys 14, 115032 (2012)], and (iii) the detrimental effect of noises on the expected outcomes. We demonstrate that, at relatively high signal-to-noise ratios and density of initial experimental points, all three built-in MATLAB interpolation functions employed in this work (i.e., Spline, Makima and $P C H I P)$ perform well in recovering the information embedded within the original sampled function; with the Spline function performing best. Whereas, by reducing either the number of initial data points or the signal-to-noise ratio, there exists a threshold below which all three functions perform poorly; with the worst performance given by the Spline function in both the cases and the least worst by the PCHIP function at low density of initial data points and by the Makima function at relatively low signal-to-noise ratios. We envisage that $\mathrm{i}-\mathrm{Rh}$ eoFT will be of particular interest and use to all those studies where sampled or time-averaged functions, often defined by a discrete set of data points within a finite time-window, are exploited to gain new insights on the systems' dynamics.
\end{abstract}

In the field of soft-matter physics, it has been shown that at thermal equilibrium the motion and the interactions between the materials' building blocks govern the linear mechanical properties of matter ${ }^{1-3}$. These can be educed via a statistical mechanics (SM) analysis of the thermally driven motion of their constituents (e.g., molecules); whose dynamics can be measured either directly (e.g., neutron spin echo ${ }^{4,5}$ ) or implicitly by means of (nano/ micro) tracers embedded into the samples ${ }^{6}$. Interestingly, the majority of these experimental methods return a measure of the materials' dynamics in an analytical form of a time-dependent exponential decay function, which in the simplest cases assumes the shape of a 'single' exponential decay $\exp \left(-t / \tau_{c}\right)$, with $\tau_{c}$ being the characteristic relaxation time of the process under investigation (e.g., the diffusion of monodisperse molecules/tracers in Newtonian fluids ${ }^{7}$ or bond percolation of transient gels $\left.{ }^{8}\right)$.

In general, for more complex systems than those just mentioned, a SM analysis of materials' thermal fluctuations may return more convoluted functions, such as a 'stretched' exponential $\exp \left(-t / \tau_{c}\right)^{\beta}$, with $\beta<1$. This is indeed a common outcome of both dynamic light scattering (DLS) ${ }^{9}$ and diffusing wave spectroscopy (DWS) ${ }^{10}$ measurements; e.g., when employed in the study of the dynamics of semi-flexible biopolymer solutions ${ }^{11}$, for which the high-frequency mechanical properties are expected to show a frequency $(\omega)$ dependency of the linear viscoelastic (LVE) moduli proportional to $\omega^{\beta}$, with $\beta=0.75^{12}$. By increasing systems' complexity, such as in the field of polymer physics, a SM analysis of the shear component of the stress tensor allows to evaluate the timedependent materials' shear relaxation modulus $G(t)^{13}$. This is often a multi-modal decay function (i.e. characterised by multiple relaxation times) that embodies, without disclosing at once, the full frequency spectrum of the materials' LVE properties. These are instead fully revealed by the frequency-dependent materials' complex shear modulus $G^{*}(\omega)$, which is a complex number whose real and imaginary parts provide quantitative information on the elastic and viscous nature of the material, respectively ${ }^{14}$. Notably, these two moduli are in principle simply related to each other by means of the Fourier transform of the time derivative of $G(t)$, whose computation given

${ }^{1}$ Division of Biomedical Engineering, James Watt School of Engineering, University of Glasgow, Glasgow G12 8LT, UK. ${ }^{2}$ SUPA, School of Physics and Astronomy, University of Glasgow, Glasgow G12 8QQ, UK. ${ }^{\square}$ email: Manlio.Tassieri@glasgow.ac.uk 
a discrete set of data is at the heart of this article. A similar issue is encountered in the field of microrheology ${ }^{15}$, where in the particular case of measurements performed with optical tweezers, a SM analysis of the trajectory of an optically trapped particle suspended into a complex fluid may return the particle normalised position autocorrelation function $A(\tau)$, or equivalently its normalised mean square displacement $\Pi(\tau)=1-A(\tau)$ (where $\tau$ is the lag-time or time interval), whose temporal form is a generic decay (or growth) function governed by the frequency-dependent linear viscoelastic properties of the suspending fluid ${ }^{16}$.

Interestingly, a common feature for all the above mentioned time-dependent functions is that they are evaluated for a discrete number of timestamps, within a finite observation time window. Yet, one of the aims of most of the studies where they are employed is often to evaluate the 'continuous' frequency spectrum of the system, over the widest range of experimentally accessible frequencies. Thus the need of an effective Fourier transform algorithm to translate the information embedded into a generic time-dependent sampled function into those equipollent, but more explicit, in the frequency-domain. This is a non-trivial task ${ }^{17}$, and has driven scientists to overcome such an issue by fitting the experimental data by means of a generalized Maxwell model (i.e., a finite sum of weighted single exponentials, each identifying a characteristic relaxation time of the system), which has a straightforward Fourier transform, but may potentially interpret the data ${ }^{18}$. Remarkably, an effective solution to this issue has been presented by Evans et al. ${ }^{17}$ and its efficacy has been augmented by Tassieri et al. ${ }^{19}$ by means of a "virtual oversampling" procedure that involves first a numerical interpolation between experimental data points by using a standard non-overshooting cubic spline function, and then generating a new, over-sampled data set, by sampling the interpolating function at a number of equally spaced points on a logarithmic time scale. The effectiveness of this method has been validated for a variety of applications within the fields of rheology and microrheology ${ }^{17,19}$; however, its general validity has been not fully exploited yet.

In this work, we have implemented the analytical method developed by Evans \& Tassieri into a open-access MATLAB code named "i-RheoFT" (allowing its use to a broad audience, see SI) and investigated its accuracy as function of three important experimental factors that are often overlooked in many applications: (i) the density of initial experimental points (DIP) describing a generic time-dependent function (i.e., the sampled function), (ii) the interpolation function used to implement the virtual oversampling procedure, and (iii) the destructive effects on the expected outcomes due to the presence of (white) noise.

As we shall demonstrate, the relative value of the first parameter plays a crucial role in the quality of the outputs of all those experimental methods (such as DLS, DWS and NSE) where data are acquired at high frequencies (e.g., at $10^{7} \mathrm{~Hz}$ or at $10^{9} \mathrm{~Hz}$ in the case of DLS and NSE measurements, respectively) and stored in the form of time-averaged functions, which are often evaluated on-the-fly by means of fast correlators. These correlation functions are commonly evaluated only for a relatively 'small' number of lag-times within a finite time window, often spanning several decades (e.g., from $10^{-7} \mathrm{sec}$ to $10^{2} \mathrm{sec}$ in the case of DLS measurements); thus avoiding the risk of clogging the machines' internal random-access memory (RAM) after a few seconds of measurement duration. The investigation of the second point has been driven by the fact that a few research groups have implemented the oversampling procedure by using different interpolation functions ${ }^{20,21}$ than the one employed in the original work ${ }^{19}$. Therefore, here we have compared the effectiveness of the following three interpolation functions already built-in MATLAB: a cubic spline data interpolation (Spline) ${ }^{22}$ (as the one used in ${ }^{19}$ ), a modified Akima piecewise cubic Hermite interpolation (Makima) ${ }^{23}$ and Piecewise Cubic Hermite Interpolating Polynomial $(\mathrm{PCHIP})^{24}$. Notably, we can anticipate that, at relatively high DIP values and signal-to-noise ratios (SNR), all three of the above mentioned interpolation methods recover the information embedded into the sampled function to a high degree of fidelity, allowing the evaluation of its Fourier transform without a significant loss of information. Whereas, at relatively low values of either of DIP or SNR, the same is not true and a clear discrimination between their efficacy is achieved in both the time- and the frequency-domains.

\section{Theoretical background}

Fourier transform of raw data. In the digital era, continuous data storing does not exist and signals are stored at a finite acquisition rate (AR), whatever fast this process could be. Therefore, measurements are represented by a finite sequence of points, often equally spaced in time and rarely acquired at time intervals equally spaced on a logarithmic scale. Nonetheless, a logarithmic timestamp is often used in post-acquisition storing procedures, such as those employed in the study of fast quasi-stochastic phenomena, for which (i) a high AR is necessary and (ii) prolonged measurements are mandatory because of statistical principles. However, these two requirements would place a high demand on the RAM capacity of any machine. Thus, the common use of correlators to evaluate on-the-fly a correlation function of the acquired signal for a finite set of lag-times often logarithmically spaced within a defined time window that spans several decades; yet, without a need of storing the raw data.

Interestingly, the discrete nature of measurements has revealed to be a hurdle to overcome in many applications, especially for those where a Fourier transform is involved; simply because the latter is a linear (integral) operator that requires the integrating function to be defined $\forall t \in]-\infty,+\infty[$, and not just for a finite set of timestamps. This is equally true for all those processes where the integrating function is defined only for positive times (i.e., $\forall t \in\left[0^{+},+\infty[\right.$ ) and it is (or assumed to be) identically equal to zero $\left.\forall t \in]-\infty, 0^{-}\right]$because of causality; such as in the studies of materials' relaxation processes after they have been subjected to either a finite deformation or a constant stress (creep), both applied within a small time interval $(\epsilon)^{14,17}$. In this regard, an analytical procedure for the evaluation of the Fourier transform of any generic function sampled over a finite time window has been proposed by Evans et al. ${ }^{17}$ to convert creep compliance $J(t)$ (defined as the ratio between the material strain $\gamma(t)$ and the applied constant stress) into $G^{*}(\omega)$ directly, without the use of Laplace transforms or fitting functions. This method is based on the interpolation of the finite data set by means of a piecewiselinear function. In particular, the general validity of the proposed procedure makes it equally applicable to find 
the Fourier transform $\hat{g}(\omega)$ of any time-dependent function $g(t)$ that vanishes for negative $t$, sampled at a finite set of data points $\left(t_{k}, g_{k}\right)$, where $k=1 \ldots N$, which extend over a finite range, and need not be equally spaced ${ }^{17}$ :

$$
\begin{aligned}
& -\omega^{2} \hat{g}(\omega)=i \omega g(0)+\left(1-e^{-i \omega t_{1}}\right) \frac{\left(g_{1}-g(0)\right)}{t_{1}}+ \\
& +\dot{g}_{\infty} e^{-i \omega t_{N}}+\sum_{k=2}^{N}\left(\frac{g_{k}-g_{k-1}}{t_{k}-t_{k-1}}\right)\left(e^{-i \omega t_{k-1}}-e^{-i \omega t_{k}}\right)
\end{aligned}
$$

where $\dot{g}_{\infty}$ is the gradient of $g(t)$ extrapolated to infinite time and $g(0)$ is the value of $g(t)$ extrapolated to $t=0$ from above.

This method has been improved by Tassieri et al. ${ }^{19}$ while analysing microrheology measurements performed with optical tweezers ${ }^{15}$. The authors found that a substantial reduction in the size of the high-frequency artefacts, from which some high-frequency noise tends to spill over into the top of the experimental frequency range, can be achieved by an over-sampling technique. The technique involves first numerically interpolating between data points using a standard non-overshooting cubic spline, and then generating a new, over-sampled data set, by sampling the interpolating function at a number of equally-spaced points on either a logarithmic or a linear timescale. We remind that, over-sampling is a common procedure in signal processing and it consists of sampling a signal with a sampling frequency $f_{s}$ much higher than the Nyquist rate $2 B$, where $B$ is the highest frequency contained in the original signal. A signal is said to be oversampled by a factor of $\beta \equiv f_{s} /(2 B)^{25}$.

Interpolation functions. In this work we have employed three built-in interpolation functions listed in MATLAB: (i) Makima, (ii) Spline and (iii) Piecewise Cubic Hermite Interpolating Polynomial (PCHIP). These are fully described in Refs. ${ }^{22-24}$ and here they are briefly summarised for convenience of the reader.

The Makima algorithm for one-dimensional interpolation, also described in Ref.s ${ }^{26,27}$, is a modification to the Akima algorithm that performs cubic interpolation to produce piecewise polynomials with continuous firstorder derivatives. The algorithm preserves the slope and avoids undulations in flat regions. A flat region occurs whenever there are three or more consecutive collinear points, which the algorithm connects with a straight line. When two flat regions with different slopes meet, the modification made to the original Akima algorithm gives more weight to the side where the slope is closer to zero. This modification gives priority to the side that is closer to horizontal, which is more intuitive and avoids overshoot. Notice that, the original Akima algorithm gives equal weights to the points on both sides, thus evenly dividing the undulation.

The Spline algorithm, performs cubic interpolation to produce piecewise polynomials with continuous secondorder derivatives. The result is comparable to a regular polynomial interpolation, but is less susceptible to heavy oscillation between data points for high degrees. Still, this method can be susceptible to overshoots and oscillations between data points at long times. Interestingly, when this is compared to the Akima algorithm, the latter produces fewer undulations and is better suited to deal with quick changes between flat regions.

The PCHIP algorithm, also performs a piecewise cubic polynomial interpolation in much the same way as the Spline function just mentioned. However, they differ in one key area which is that while the Spline functions' second-order derivative is continuous, the second-order derivative for PCHIP is unlikely to be and therefore the interpolation function is shape preserving for large jumps between data points. Additionally, the non continuous nature of the second-derivative means that PCHIP has no overshoots and much lower oscillation when the data is not smooth when compared to the Spline function.

Sampled functions and the density of initial points. In order to quantify the fidelity of the above mentioned interpolation procedures in recovering the original information contained by a sampled function, we have employed them to evaluate the Fourier transform (via Eq. 1) of two functions that are similar to those often seen in optical tweezers experiments ${ }^{15,16}$ : (I) a single exponential decay function:

$$
A(t)=\exp \left(-t / \tau_{c}\right)
$$

describing the dynamics of a Maxwell fluid characterised by a single relaxation time $\tau_{c}$, as shown in Fig. 1 (top); and (II) a set of data resembling the mean square displacement of a weakly trapped probe particle suspended into a non-Newtonian fluid (Fig. 1 (bottom)), which have been evaluated by means of the following series:

$$
\Pi(q, t)=\sum_{q=1}^{\infty} \frac{1}{q^{4}}\left(1-e^{-q^{4} t}\right), \quad t \geqslant 0,
$$

where $q$ is the mode number and time is measured in units of the longest relaxation time for $q=1$. It is important to notice that Eq. (3) is a series definitely convergent, and in this work we have used its approximant $\Pi_{11}(q, t)=\sum_{q=1}^{11}\left(1-e^{-t q^{4}}\right) q^{-4}$; which provides a good estimation of the series $\forall t$. This has been corroborated by evaluating the incremental value of the mean relative error of the approximant $\Pi_{11}(q, t)$ when compared to the approximants $\Pi_{100}(q, t)$ and $\Pi_{200}(q, t)$, for $t \in\left[10^{-2}, 10^{2}\right]$; which results in being as low as $0.0000287 \%$.

Interestingly, the Fourier transform of the time derivative of both the functions described by Eqs. (2) and (3) can be calculated analytically and therefore an exact expression of the related complex moduli (i.e., $G_{A}^{*}(\omega)$ and $G_{\Pi}^{*}(\omega)$, describing the viscoelastic properties of the suspending fluids) can be derived for both of them ${ }^{15,16,18}$ : 

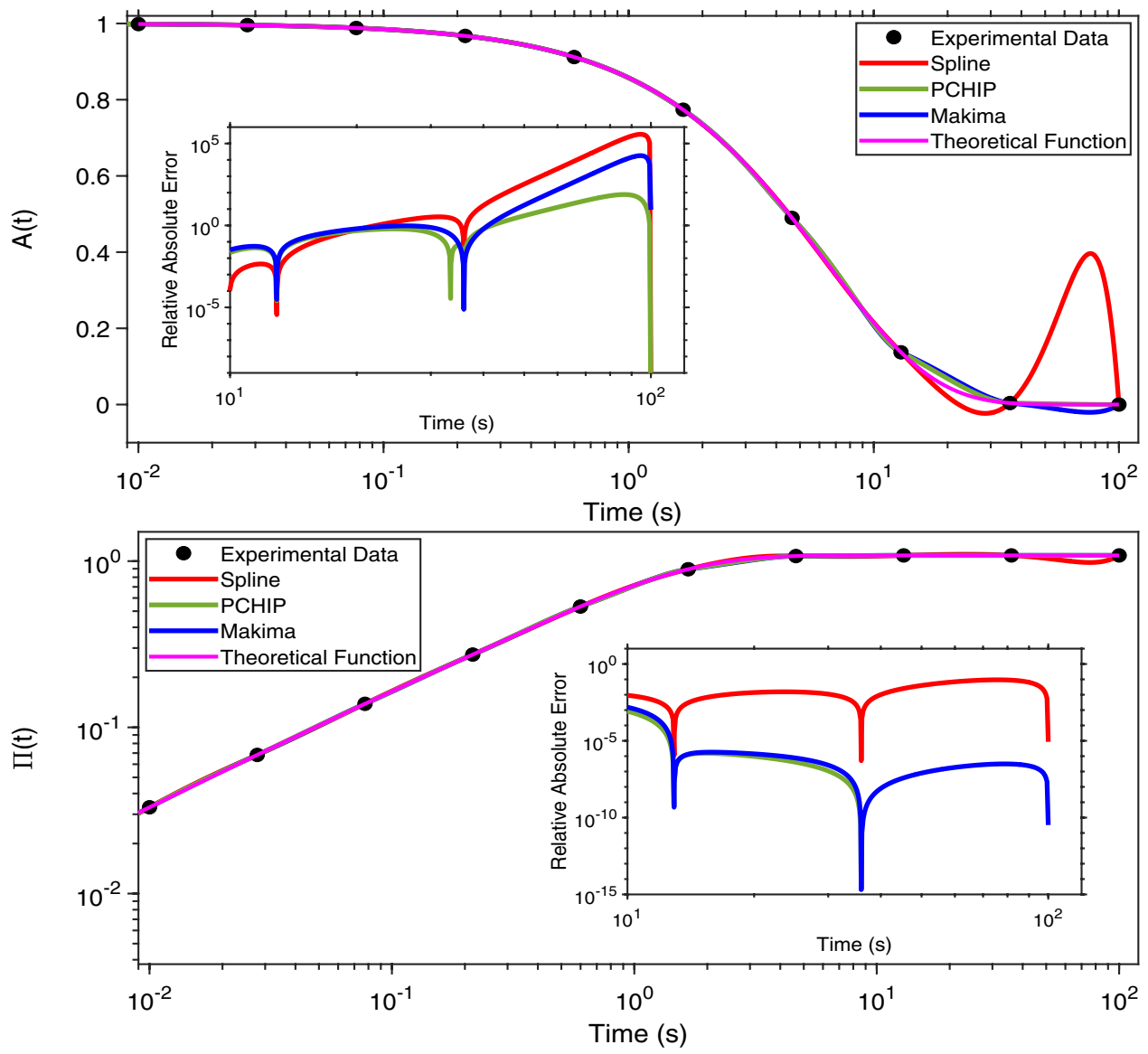

Figure 1. (Top) A single exponential decay function vs. time, representing the relaxation modulus of a single mode Maxwell fluid. (Bottom) A generic function resembling the normalised mean square displacement vs. time of an optically trapped particle suspended into a non-Newtonian fluid. Equations (2) and (3) are represented by a finite number of 'sampled' points and a continuous (pink) line. The points are also interpolated by means of three MATLAB built-in interpolation functions: Spline, PCHIP and Makima. The insets show the relative absolute error of each interpolation function with respect of either of Eqs. (2) and (3), as calculated using Eq. (7). The time window of the inset encompasses the final three points of the main graph, where the relative error is at its highest.

$$
G_{A}^{*}(\omega)=i \omega \hat{A}(\omega)=\frac{\left(\omega \tau_{c}\right)^{2}}{1+\left(\omega \tau_{c}\right)^{2}}+i \frac{\omega \tau_{c}}{1+\left(\omega \tau_{c}\right)^{2}}
$$

and

$$
G_{\Pi}^{*}(\omega)=[i \omega \hat{\Pi}(\omega)]^{-1}=\left[\sum_{q=1}^{11} \frac{\left(q^{4}-i \omega\right)}{\left(q^{8}+\omega^{2}\right)}\right]^{-1}
$$

where $\hat{A}(\omega)$ and $\hat{\Pi}(\omega)$ are the Fourier transforms of the functions described by Eqs. (2) and (3), respectively. It follows that, Eqs. (4) and (5) can each act as a reference for a quantitative evaluation of the errors generated during the Fourier transform of a discrete set of data representing either of Eqs. (2) or (3), as function of both the density of initial points (DIP) and the oversampling factor $(\beta)$. However, while the latter is a well known parameter in signal processing and would not affect the effectiveness of Eq. (1) for relatively high values of $\beta^{19}$ (here it is kept constant to 50), DIP is introduced in this work to inform the scientific community of its relevance when modelling or interpreting a discrete set of data by means of a continuous interpolation function:

$$
D I P=\frac{\log _{10}\left(N_{e}\right)}{\log _{10}\left(t_{N} / t_{1}\right)}
$$

where $N_{e}$ is the number of experimental data points within the explored time window, which extends from a lower end equal to $t_{1}$ to a maximum time equal to $t_{N}$. Therefore, a function sampled at a few MHz over a time window spanning from $t_{1}=10^{-7}$ s to $t_{N}=10$ s would have DIP $=1$ if $N_{e}$ were equal to $10^{8}$. Interestingly, this is not the case for the majority of the experimental techniques mentioned in the introduction (e.g. DLS, DWS, 
etc.), for which the technological constrain dictated by the finite RAM of the instruments is compensated by the adoption of correlators that convolve the high-speed acquired data into a finite set of averaged values calculated for a relatively small number of lag-times. Thus, returning a DIP value often much smaller than 1; which, as we shall demonstrate below, may lead to erroneous outcomes, especially at relatively low DIP values.

\section{Results and discussion}

Let us start by considering both Eqs. (2) and (3) sampled at a relatively low acquisition rate, as shown in Fig. 1. Both the functions are represented by 10 experimental points equally spaced on a logarithmic scale (black dots) and a continuous (pink) line, within a time window ranging from $10^{-2}$ to $10^{2} \mathrm{~s}$; which implies a DIP= $1 / 4$.

The experimental data have been interpolated by using the three MATLAB built-in functions mentioned above and compared with the theoretical curve. At a glance, from Fig. 1 it is possible to see the detrimental effect caused by a relatively low DIP value on all three interpolation processes, especially at large lag-times, where the Spline function performs worse. In order to quantify such a discrepancy, we have evaluated the relative absolute error $(\operatorname{RAE}(t))$ of the three interpolation functions $\left(g_{I}(t)\right)$ with respect to the theoretical functions computed by means of Eqs. (2) and (3):

$$
R A E(t)=\frac{\left|g_{I}(t)-A(t)\right|}{A(t)} \text { or } R A E(t)=\frac{\left|g_{I}(t)-\Pi(t)\right|}{\Pi(t)}
$$

The insets in Fig. 1 show the $\operatorname{RAE}(t)$ of the three interpolation functions, with a focus in the time window ranging from $10^{1} \mathrm{~s}$ to $10^{2} \mathrm{~s}$, where the error is at its highest. Interestingly, in the case of the single exponential decay, all three RAEs increase rapidly by almost ten decades across the explored time window, with the Spline function returning the highest error. A similar outcome can be seen in the case of $\Pi(t)$, where both PCHIP and Makima interpolation functions perform significantly better than the Spline function, returning a RAE $(t)$ at long times almost five decades smaller. Notice that, the minima in the RAE $(t)$ are due to the inherent nature of interpolation functions to pass through each experimental data point; a condition that is not guaranteed by any fitting procedure.

In order to investigate the fidelity of the interpolation process as function of DIP, we have evaluated the mean relative absolute error (MRAE) of the interpolating functions for $N_{e}$ varying from 10 to $10^{4}$, which implies a DIP ranging from $1 / 4$ to 1 (see Fig. 2). The MRAE is defined as follows:

$$
M R A E=\frac{100}{N} \sum_{n=1}^{N} \frac{\left|g_{I}(n)-A(n)\right|}{A(n)}
$$

where $N$ is the number of points at which all the functions are evaluated within the experimental time window $\left[10^{-2}, 10^{2}\right]$; in this study $N=10^{4}$. Notice that, a similar expression to Eq. (8) can be written in terms of $\Pi(t)$. From Fig. 2 it is interesting to note that, at relatively low DIP values, all three functions perform poorly; with the Spline function performing worst and the PCHIP returning the lowest error in both the cases; yet higher than $10^{3} \%$ in the case of the single exponential decay (Fig. 2, top). We argue that, the better fidelity shown by the PCHIP function can be attributed to the non continuous nature of the second-derivative of its interpolation algorithm, which prevents from overshoots and returns much lower oscillation in case of large jumps between data points (i.e., at low DIP) when compared to the Spline function.

Notably, for DIP values approaching 1, all three interpolation functions successfully recover the sampled functions to a high degree of accuracy, returning MRAE values lower than $10^{-5} \%$ for the case of the single exponential decay. In this case, from a practical point of view, it is worth noting that the MRAE of all three interpolation functions falls below $1 \%$ for DIP values higher than circa 0.56 , which implies a minimum number of 174 initial sampled points within the explored time window. Interestingly, when this outcome is applied for instance to DLS measurements (for which the experimental time window spans from $10^{-7} \mathrm{sec}$ to $10^{2} \mathrm{sec}$ ), a DIP of 0.56 would imply a minimum number of $\approx 10^{5}$ initial timestamps (or lag-times); a condition never met in real experiments, where instead a DIP of circa 0.25 is commonly found. In the case of the NMSD (see Fig. 2, bottom), at low DIP values, both PCHIP and Makima start with a MRAE smaller than $0.1 \%$, while the Spline function starts with a MRAE value of circa 4\%; thus confirming its poor performance at relatively low DIP values. Nonetheless, when comparing the errors generated by the interpolation procedures for the two cases of study, it is striking the different behaviour of their MRAE curves, which differ from each other by almost five orders of magnitude in opposite direction at the extremes of the explored range of DIP values.

Let us now investigate how the error propagates into the frequency-domain as function of both the chosen interpolation algorithm and the DIP, when performing the Fourier transform by means of the Evans \& Tassieri's method. In Fig. 3, the viscoelastic moduli evaluated by means of Eqs. (4) and (5) are drawn together with those derived by Fourier transforming the interpolation functions shown in Fig. 1 for the case of DIP $=1 / 4$.

From Fig. 3, it is evident the detrimental effects of using a relatively low DIP value for determining the dynamic information embedded within the sampled functions. In particular, as discussed earlier, the Spline function carries the biggest error because of its poor performance in resembling the sampled functions at long times, which translates into artefacts in the low-frequency behaviour of both the moduli. Notably, these artefacts are significantly reduced in the case of Makima and even further in the case of PCHIP, which is the one that performs best at low DIP values. In Fig. 3 we also report the viscoelastic moduli evaluated in the case of DIP $=1$ as a means of comparison. The inset of Fig. $3 \mathrm{~h}$ highlights the relatively small divergence of the elastic modulus from the expected value at high frequencies shown by all three of the interpolation functions. We argue that this (small) discrepancy is caused by the different constrains on the first and second derivatives adopted by the three 

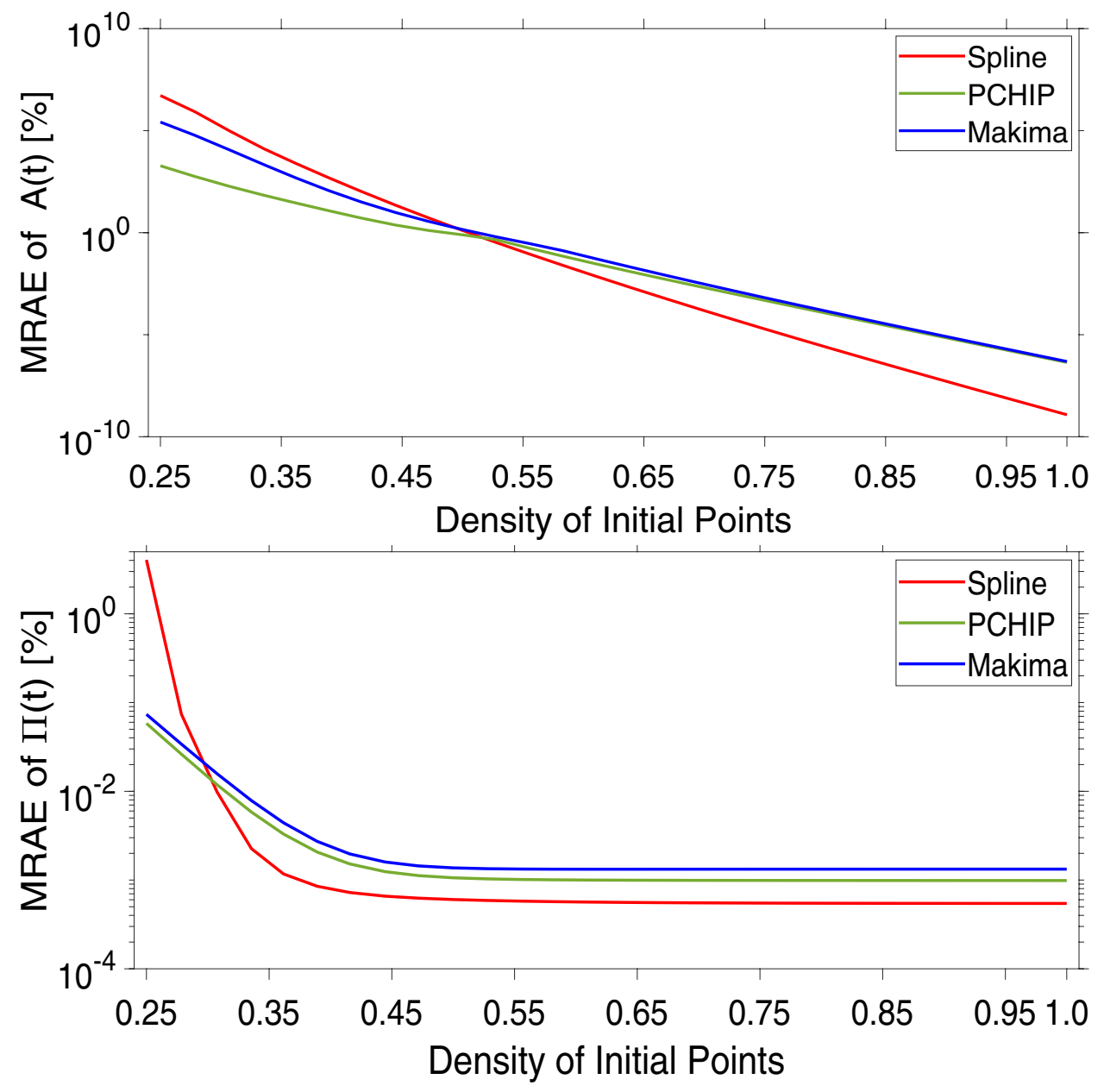

Figure 2. Mean relative absolute error (MRAE) vs. the density of initial experimental points (DIP) of the three MATLAB built-in interpolation functions: Spline, PCHIP and Makima. (Top) The MRAE is evaluated with respect to Eq. (2). (Bottom) The MRAE is evaluated with respect to Eq. (3).

interpolation algorithms to model the sampled function at the boundaries of the experimental time window; i.e, here within the time-gap occurring between the first experimental point at $t_{1}$ and the asymptotic one at $t=0$.

In order to quantify these discrepancies, we have evaluated the MRAE of both the real and imaginary parts (i.e., $G_{I}^{\prime}(\omega)$ and $G_{I}^{\prime \prime}(\omega)$ ) of the calculated complex modulus with respect to their expected values (i.e., $G_{A}^{\prime}(\omega)$ and $G_{A}^{\prime \prime}(\omega), G_{\Pi}^{\prime}(\omega)$ and $\left.G_{\Pi}^{\prime \prime}(\omega)\right)$ obtained from Eqs. (4) and (5), respectively:

$$
M R A E=\frac{1}{N_{\omega}} \sum_{n=1}^{N_{\omega}} \frac{\left|G_{I}^{\prime}\left(\omega_{n}\right)-G_{A}^{\prime}\left(\omega_{n}\right)\right|}{G_{A}^{\prime}\left(\omega_{n}\right)}
$$

where $n=1 \ldots N_{\omega}$ is the number of frequencies at which Eq. (9) is evaluated (here $N_{\omega}=200$, with $\omega_{n}$ equally spaced on a logarithmic scale ranging from $10^{-2}$ to $10^{2} \mathrm{~Hz}$ ). A similar expression can be written for the viscous modulus, with $G_{I}^{\prime}\left(\omega_{n}\right)$ and $G_{A}^{\prime}\left(\omega_{n}\right)$ replaced by $G_{I}^{\prime \prime}\left(\omega_{n}\right)$ and $G_{A}^{\prime \prime}\left(\omega_{n}\right)$, and for Eq. (5) by replacing $G_{A}^{*}\left(\omega_{n}\right)$ with $G_{\Pi}^{*}\left(\omega_{n}\right)$, respectively. In particular, we have evaluated the MRAE for DIP values ranging from 1/4 to 1 ; and the results are reported in Fig. 4 for both the sampled functions.

From Fig. 4 top, it can be seen that (i) at relatively low DIP values (i.e., for DIP $\lesssim 0.33$ ) both the interpolation functions PCHIP and Makima perform significantly better than the Spline one; which, (ii) for DIP $>0.33$ returns relatively lower values of the MRAE for both the moduli. Interestingly, for DIP $>0.9$ the MRAE reaches a plateau value for both the moduli and all the interpolation functions; with the MRAEs of the elastic modulus showing an identical value for all three interpolation functions starting from DIP $=0.75$. Moreover, it can be seen that for $0.33<$ DIP $<0.9$ both PCHIP and Makima return a MRAE of the viscous modulus much higher than for the elastic one, which is actually comparable to the MRAE of both the moduli obtained by means of a Spline interpolation. From Fig. 4 top, we can assert that, all the three interpolation functions provide a MRAE of both the moduli smaller than $1 \%$ for DIP $>0.4$, which implies the need of a minimum number of initial data points of circa 40 within the explored time window. 

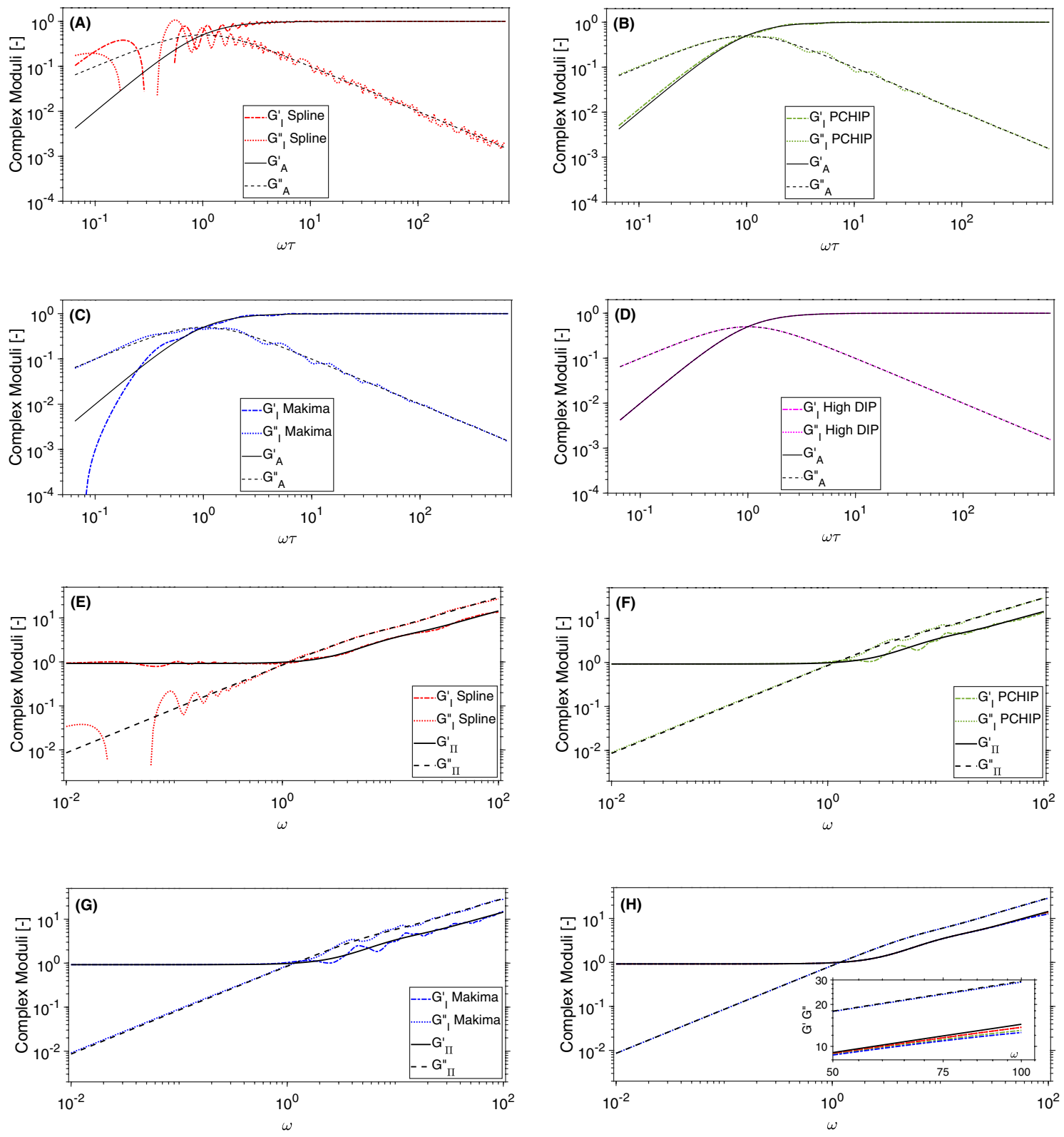

Figure 3. Comparison between the frequency-dependent complex moduli obtained via Eq. (4) and those evaluated by Fourier transforming via Eq. (1) the interpolations functions shown in Fig. 1 for DIP $=1 / 4$ in (A, $\mathbf{B}, \mathbf{C}, \mathbf{E}, \mathbf{F}, \mathbf{G})$ and for DIP $=1$ in $(\mathbf{D}, \mathbf{H})$.

From Fig. 4 bottom, it can be seen that (i) at relatively low DIP values (i.e., for DIP $\lesssim 0.28$ ) both the interpolation functions PCHIP and Makima return a similar value of the MRAE (i.e. lower than 5\%) for both the moduli; whereas, the Spline function returns a similar value of the MRAE only for the elastic modulus, while the MRAE for the viscous modulus goes up to a value of $50 \%$ at DIP $=0.25$. Interestingly, for DIP $>0.5$ the MRAE of both the viscoelastic moduli reach similar constant values for all the interpolation functions. Specifically, the MRAEs of the elastic modulus is of the order of $1 \%$ and the MRAEs of the viscous modulus is of the order of $0.1 \%$. Notably, also in this case, the Spline function returns overall a lower (and constant) MRAE than both PCHIP and Makima for DIP $>0.4$.

Let us now investigate how the presence of (white) noise impacts on the effectiveness of the analytical method developed by Evans \& Tassieri, when the above mentioned interpolation functions are employed to analyse both Eqs. (2) and (3), sampled at a fixed acquisition rate and for which the amplitude of the added noise is varied to explore a range of signal-to-noise ratios (SNR) spanning from $1 \mathrm{~dB}$ to $350 \mathrm{~dB}$. The latter has been calculated by using the following equation:

$$
S N R=\left(\frac{A_{\text {signal }}}{A_{\text {noise }}}\right)^{2},
$$



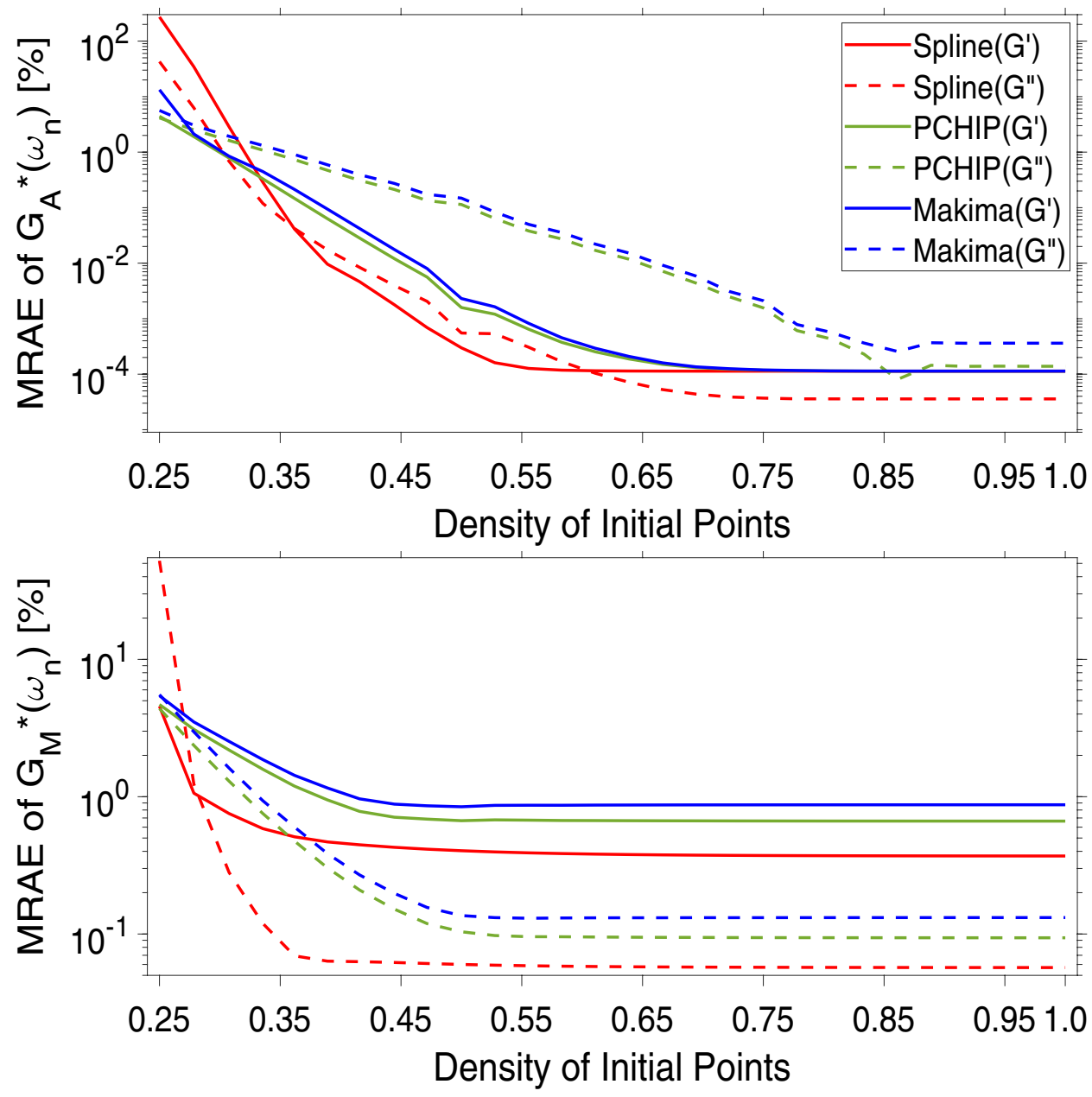

Figure 4. Mean relative absolute error (MRAE) of the frequency-dependent complex moduli determined by Fourier transforming (via Eq. 1) the interpolation functions shown in Fig. 1 (top \& bottom) for DIP values ranging from $1 / 4$ to 1 .

where $A_{\text {signal }}$ and $A_{\text {noise }}$ are the root mean square amplitudes of the signal and the noise, respectively.

As a means of discussion, in Fig. 5 are reported both Eqs. (2) (top) and (3) (bottom) drawn as continuous (pink) lines by using $10^{4}$ experimental points linearly spaced within a time window of $\left[10^{-2}, 10^{2}\right]$ sec (i.e., DIP $=1$ ), to which a random white noise having a $\mathrm{SNR}=50$ has been added. The resulting 'noisy' functions have been then interpolated by means of all three of the above mentioned MATLAB built-in interpolation functions. From Fig. 5, and further elucidated hereafter, it can be seen that, at relatively low SNR, the different nature of the interpolation functions can lead to very large deviations from the expected values within the time window $\left[0, t_{1}\right]$, where $t_{1}=1 / A R$. This is especially true in the case of the Spline function, as shown by the insets of Fig. 5 , both at top and bottom.

In contrast, due to its non-continuous nature of the second derivative that prevents overshoots, the PCHIP algorithm performs better than both Spline and Makima functions. Notably, as we shall demonstrate below, these deviations are the major source of error at high frequencies when performing the Fourier transform via Eq. (1), which otherwise reveals to be almost unaffected at low frequencies by the presence of the noise themselves; as shown in Fig. 6, but also supported by the experimental evidences reported in Figures 7 and 8 of Ref. ${ }^{14}$. Here, Fig. 6 shows a comparison between the viscoelastic moduli calculated via Eqs. (4) (top) and (5) (bottom) and those evaluated by Fourier transforming via Eq. (1) the interpolations functions shown in Fig. 5. From Fig. 6 it is clear that the high frequency noise caused by the 'miss modelling' of the short time (i.e., $\forall t \in] 0, t_{1}[$ ) behaviour of the experimental data tends to spill over into the top of the experimental frequency range, with the moduli evaluated either via PCHIP or Makima adhering most to the exact solutions, especially at relatively low frequencies; whereas the Spline function performs worse over the whole frequency range. Interestingly, also in this case, for a given set of experimental data, there exists a threshold value of the SNR above which all the three interpolation functions allow an accurate estimation of the Fourier transform.

In order to identify the threshold value for each interpolation method applied to both Eqs. (2) and (3) (with DIP = 1), we have evaluated the MRAE of the transformed data (i.e., Eqs. 9) as function of the SNR; with the latter ranging from $1 \mathrm{~dB}$ to $350 \mathrm{~dB}$. The results are reported in Fig. 7 for both the sampled functions shown in 


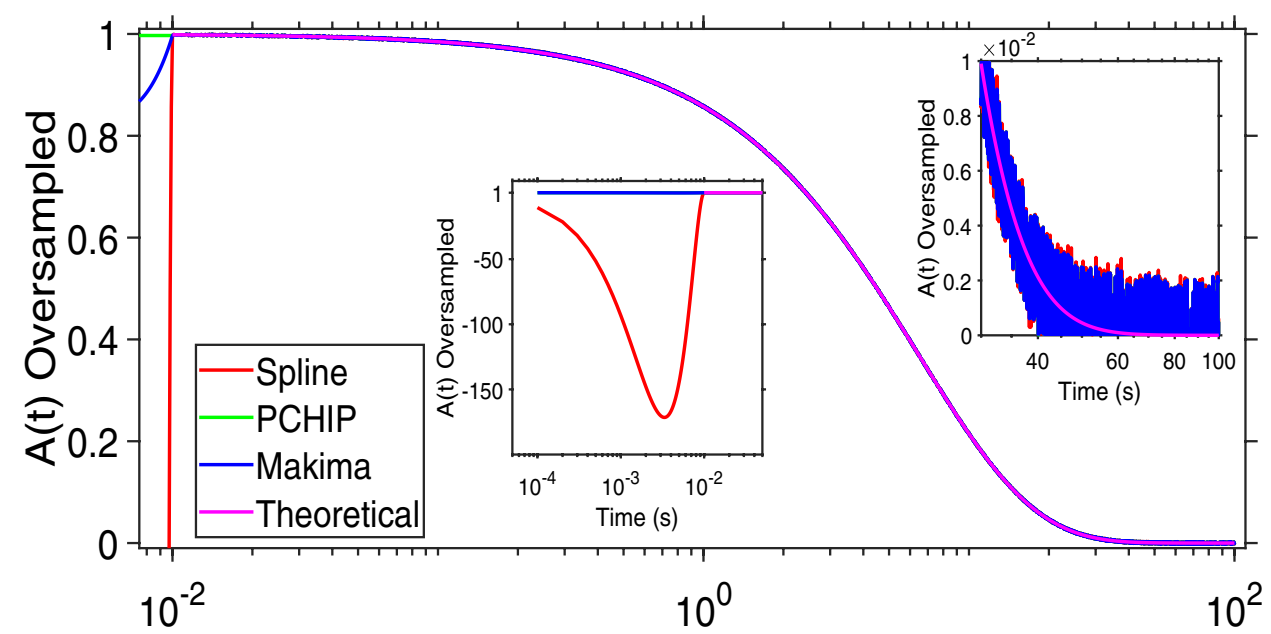

Time (s)

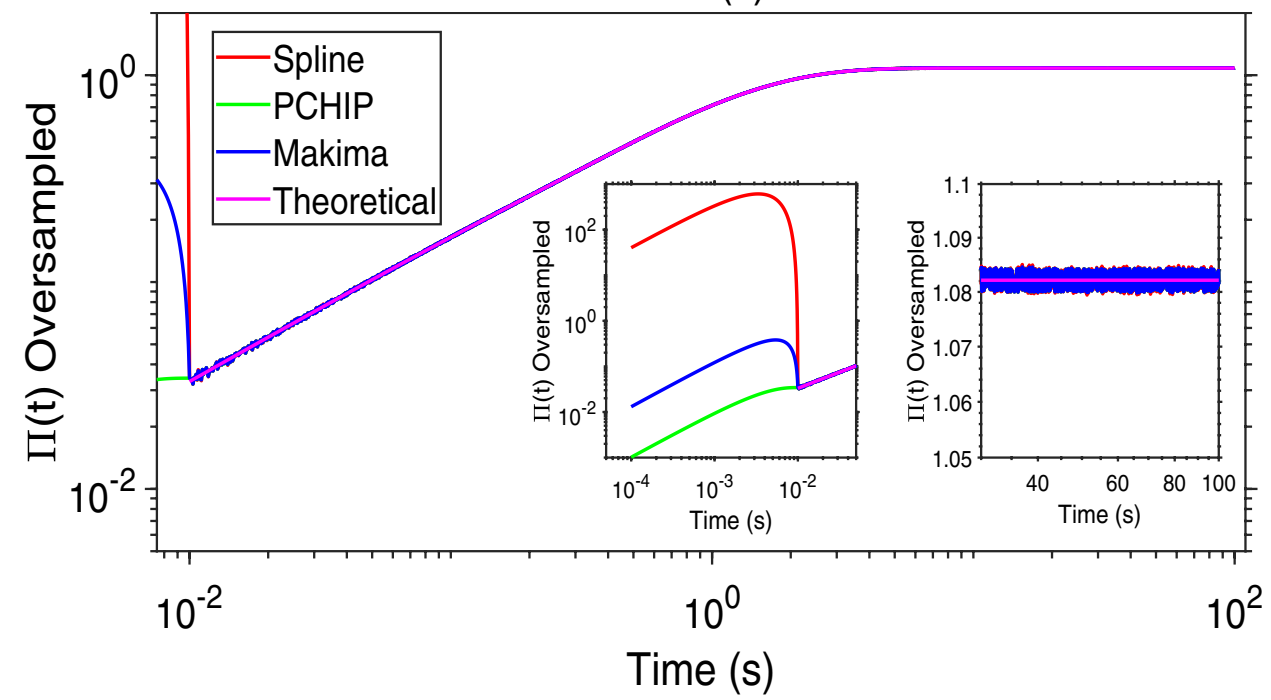

Figure 5. (Top) Eq. (2) and (bottom) Eq. (3) drawn as continuous (pink) lines by using $10^{4}$ experimental points linearly spaced in time. A white noise having a $\mathrm{SNR}=50$ is added to the experimental data, which are then interpolated by means of three MATLAB built-in interpolation functions: Spline, PCHIP and Makima. The insets highlight the detrimental effects on the interpolation process due to the presence of noise, both at short and long time scales.

Fig. 5. From Fig. 7 top, it can be seen that at low values of SNR (i.e. $\lesssim 50 \mathrm{~dB}$ ) all of the interpolation functions perform poorly, with a MRAE as large as $10^{8} \%$ for the Spline interpolation function at SNR = 0; thus performing significantly worse than PCHIP and Makima. However, as one would expect, by increasing the SNR to a relatively high value (here above $\sim 170 \mathrm{~dB}$ ) the MRAEs of the moduli associated with each interpolation function fall below $1 \%$ and asymptotically approach the values presented in Fig. 4 for DIP $=1$. Interestingly, from Fig. 7, one could argue that, in presence of noise, the interpolation processes performed by PCHIP and Makima work best, when compared to the Spline. However, in response it must be highlighted that, in real experiments, it is rare to process data with low SNR; such as those reported in the insets of Fig. 5, where both the Eqs. (2) and (3) are drawn with a $\mathrm{SNR}=50$, which would commonly be discarded as 'noisy measurements'.

\section{Conclusion}

In this article we have presented an open-access code named i-RheoFT that allows to evaluate the Fourier transform of any generic time-dependent function that vanishes for negative times, sampled at a finite set of data points that extend over a finite range, and need not be equally spaced. The analytical method that underpins this code has been originally introduced by Evans \& Tassieri ${ }^{17,19}$ in the form of an open-access LabVIEW executable specialised for the analysis of microrheology measurements performed with optical tweezers. Here we expand the range of its potential applications by implementing it into an open-access MATLAB code, with the aim of reaching a broader audience and encouraging its exploitation in a variety of applications.

The effectiveness of i-RheoFT has been corroborated here by evaluating the Fourier transform of two generic functions having a known analytical expression of their Fourier transforms. Moreover, the analytical method 

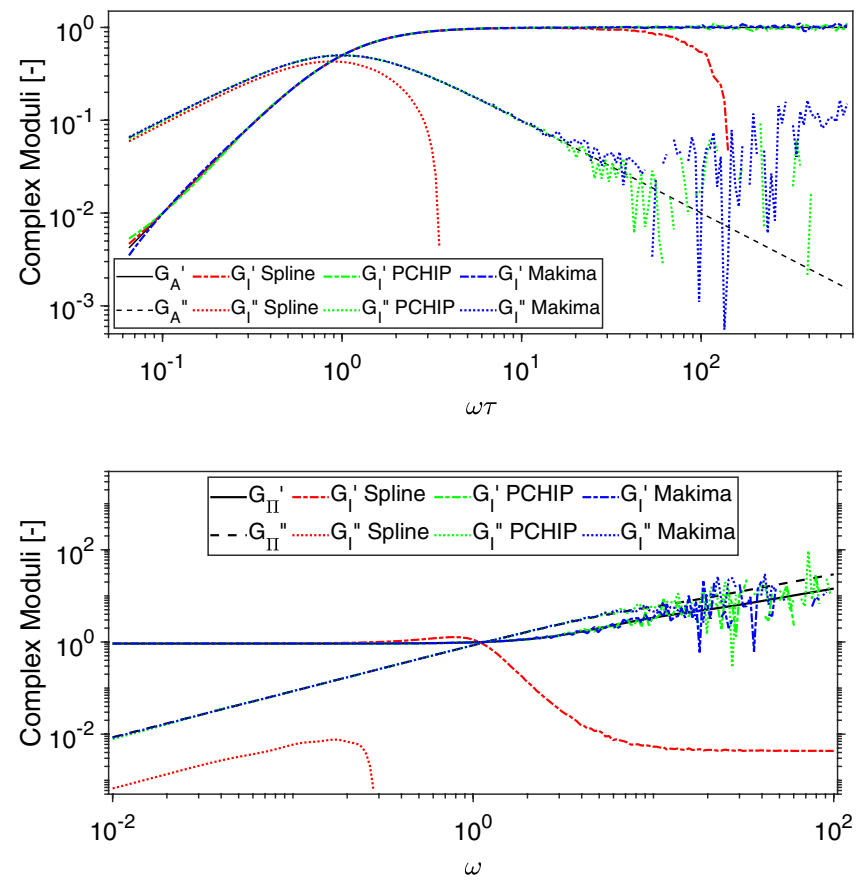

Figure 6. Comparison between the frequency-dependent complex moduli obtained via Eqs. (4) (top) and (5) (bottom) and those evaluated by Fourier transforming via Eq. (1) the interpolations functions shown in Fig. 5.
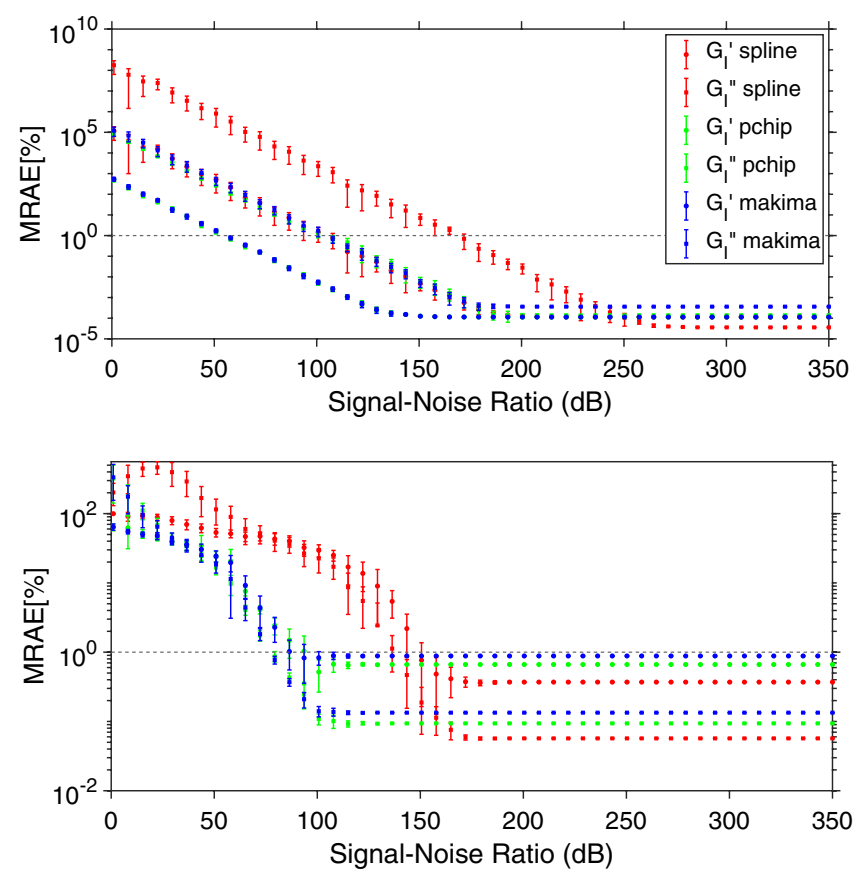

Figure 7. Mean relative absolute error (MRAE) of the frequency-dependent complex moduli determined by Fourier transforming (via Eq. 1) the interpolation functions shown in Fig. 5 (top \& bottom respectively) for SNR values ranging from 1 to $350 \mathrm{~dB}$. The error bars represent one standard deviation of uncertainty calculated over ten repeats.

has been tested as function of three important experimental parameters: (i) the 'density of initial experimental points' (DIP) describing the sampled function; (ii) the interpolation algorithm used to perform the "virtual oversampling" procedure introduced by Evans \& Tassieri, which here is achieved by means of the following three built-in MATLAB functions: Spline, Makima and PCHIP; and (iii) the destructive effects on the expected outcomes due to the presence of (white) noise. 
The outcomes of this study reveal that, at relatively high DIP values and signal-to-noise ratios, all three interpolation functions perform well in recovering the original information of the sampled function, with the Spline function always performing best. Whereas, by reducing either the number of initial data points or the signal-to-noise ratio, there exists a threshold value below which all three functions perform poorly, with the Spline function always returning the highest error of all three functions.

Therefore, we envisage that these results and i-RheoFT will be of particular interest and use to all those (experimental and simulation) studies where fast streams of acquired data are processed on-the-fly to build time-averaged functions, which are often defined by a finite number of data points over a limited time window spanning several decades; as for instance, in the cases of diffusing wave spectroscopy and dynamic light scattering measurements.

Received: 9 March 2021; Accepted: 22 November 2021

Published online: 15 December 2021

\section{References}

1. Ferry, J. Viscoelastic Properties of Polymers (Wiley, 1980).

2. Rubinstein, M. Polymer Physics (Oxford University Press, 2003).

3. McLeish, T. C. B. Tube theory of entangled polymer dynamics. Adv. Phys. 51(6), 1379-1527 (2002).

4. Ewen, B. Neutron Spin Echo Spectroscopy, Viscoelasticity, Rheology (Springer, 1997).

5. Richter, D., Monkenbusch, M., Arbe, A. \& Colmenero, J.. Neutron Spin Echo in Polymer Systems (Springer, 2005).

6. Rizzi, L. G. \& Tassieri, M. Microrheology of biological specimens. Encycl. Anal. Chem., 1-24, (2018).

7. Waigh, T. A. Advances in the microrheology of complex fluids. Rep. Progress Phys. 79(7), 074601 (2016).

8. Lodge, J. F. M. \& Heyes, D. M. Transient colloidal gels by brownian dynamics computer simulation. Phys. Chem. Chem. Phys. 1(9), 2119-2130 (1999).

9. Berne, B. Dynamic Light Scattering: With Applications to Chemistry, Biology, and Physics (Dover Publications, 2000).

10. Pine, D. J., Weitz, D. A., Chaikin, P. M. \& Herbolzheimer, E. Diffusing wave spectroscopy. Phys. Rev. Lett. 60(12), 1134-1137 (1988).

11. Tassieri, M., Evans, R. M. L., Barbu-Tudoran, L., Khaname, G. N., Trinick, J., \& Waigh, T. A. Dynamics of semiflexible polymer solutions in the highly entangled regime. Phys. Rev. Lett., 101(19), (2008).

12. Tassieri, M. Dynamics of semiflexible polymer solutions in the Tightly Entangled Concentration regime. Macromolecules 50(14), 5611-5618 (2017).

13. Tassieri, M., Ramírez, J., Karayiannis, N. C., Sukumaran, S. K. \& Masubuchi, Y. i-rheo GT: transforming from time to frequency domain without artifacts. Macromolecules 51(14), 5055-5068 (2018).

14. Tassieri, M. et al. i-rheo: measuring the materials" linear viscoelastic properties “in a step”!. J. Rheol. 60(4), 649-660 (2016).

15. Tassieri, M. Microrheology with Optical Tweezers: Principles and Applications (Pan Stanford Publishing, 2016).

16. Tassieri, M. Microrheology with optical tweezers: peaks \& troughs. Curr. Opin. Colloid Interface Sci. 43, 39-51 (2019).

17. Evans, R. M. L., Tassieri, M., Auhl, D., \& Waigh, T. A. Direct conversion of rheological compliance measurements into storage and loss moduli. Phys. Rev. E, 80(1), (2009).

18. Tassieri, M. Comment on "A symmetrical method to obtain shear moduli from microrheology" Soft Matter, 14(42):8666-8670, (2018).

19. Tassieri, M., Evans, R. M. L., Warren, R. L., Bailey, N. J. \& Cooper, J. M. Microrheology with optical tweezers: data analysis. New J. Phys. 14(11), 115032 (2012).

20. Peddireddy, K. R. et al. Unexpected entanglement dynamics in semidilute blends of supercoiled and ring dna. Soft Matter 16, 152-161 (2020).

21. Alshareedah, I., Moosa, M. M., Pham, M., Potoyan, D. A., \& Banerjee, P. R. Programmable viscoelasticity in protein-RNA condensates with disordered sticker-spacer polypeptides. Nat. Communicat. 12(1), 6620 (2021).

22. MathWorks. Spline, Cubic Spline Data Interpolation. Accessed 28 Sept 2020.

23. MathWorks. Makima, Modified Akima Piecewise Cubic Hermite Interpolation. Accessed 28 Sep 2020.

24. MathWorks. PCHIP, Piecewise Cubic Hermite Interpolating Polynomial (PCHIP). Accessed 28 Sep 2020.

25. Lathi, B. P. Linear Systems and Signals (Oxford University Press, 2005).

26. Akima, H. A new method of interpolation and smooth curve fitting based on local procedures. J. ACM 17(4), 589-602 (1970).

27. Akima, H. A method of bivariate interpolation and smooth surface fitting based on local procedures. Commun. ACM 17(1), 18-20 (1974).

\section{Acknowledgements}

This work was supported by the EPSRC CDT in "Intelligent Sensing and Measurement" (EP/L016753/1). M.T. acknowledges support via EPSRC grant "Experiencing the micro-world - a cell's perspective" (EP/R035067/1 $\mathrm{EP} / \mathrm{R} 035563 / 1-\mathrm{EP} / \mathrm{R} 035156 / 1)$.

\section{Author contributions}

M.T. conceived the method. M.G.S. implemented the MATLAB code and performed the data analysis. M.G.S., G.M.G. and M.T. contributed writing the manuscript.

\section{Competing interests}

The authors declare no competing interests.

\section{Additional information}

Supplementary Information The online version contains supplementary material available at https://doi.org/ 10.1038/s41598-021-02922-8.

Correspondence and requests for materials should be addressed to M.T.

Reprints and permissions information is available at www.nature.com/reprints. 
Publisher's note Springer Nature remains neutral with regard to jurisdictional claims in published maps and institutional affiliations.

(c) (i) Open Access This article is licensed under a Creative Commons Attribution 4.0 International License, which permits use, sharing, adaptation, distribution and reproduction in any medium or format, as long as you give appropriate credit to the original author(s) and the source, provide a link to the Creative Commons licence, and indicate if changes were made. The images or other third party material in this article are included in the article's Creative Commons licence, unless indicated otherwise in a credit line to the material. If material is not included in the article's Creative Commons licence and your intended use is not permitted by statutory regulation or exceeds the permitted use, you will need to obtain permission directly from the copyright holder. To view a copy of this licence, visit http://creativecommons.org/licenses/by/4.0/.

(C) The Author(s) 2021 